\title{
"Posterior-like" anterior per-oral endoscopic myotomy: a novel concept
}

\section{Georgios Mavrogenis, Pavlos Antoniou, loannis Tsevgas, Dimitrios Zachariadis}

Department of Gastroenterology, Mediterraneo Hospital, Athens, Greece

Peroral endoscopic myotomy (POEM) has been established as an emerging technique for the management of Achalasia. Currently, two different approaches have been described, depending on the operator's preference: with the patient in the supine position, anterior POEM is performed at the 2 o'clock position, while posterior POEM is performed at the 6 oclock position. Recent data show them to have similar efficacy $[1,2]$, suggesting less acid exposure after anterior POEM but a higher rate of mucosotomies due to the tangential approach [1]. In posterior POEM the dissection plane is more convenient; however, the pooling of liquids/blood in the 6 oclock position can be disturbing. Here we describe how to perform anterior POEM while benefiting from the advantages of posterior POEM.

Once the mucosal entry has been created, the endoscopist's body is turned towards the patient's feet, where a second monitor is placed, and the shaft of the endoscope is rotated anticlockwise (Fig. 1). In this way the axis of the tunnel is positioned at the 5-7 oclock position (Fig. 2, Video 1). Further dissection and myotomy are continued using the monitor placed by the patient's feet. So far, we have experience from 3 cases of "posterior-like" anterior POEM with a technical success rate of $100 \%$.

Video 1 Video demonstration of posterior-like anterior POEM

Department of Gastroenterology, Mediterraneo Hospital, Athens, Greece

Conflict of Interest: None

Correspondence to: Georgios Mavrogenis, Kavetsou 10, 81100

Mytilene, Greece, e-mail: mavrogenis@gmail.com

Received 23 June 2018; accepted 13 July 2018; published online 20 July 2018

DOI: https://doi.org/10.20524/aog.2018.0298

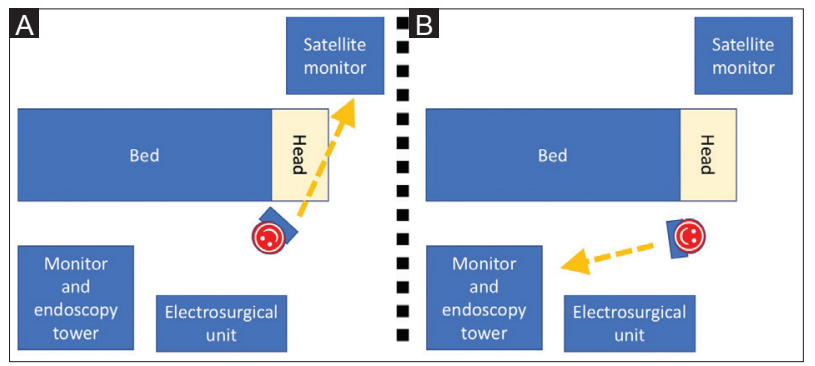

Figure 1 (A) Standard setup for anterior peroral endoscopic myotomy (POEM). (B) Setup for "posterior-like" anterior POEM. The endoscopist performs the procedure through the monitor placed by the patient's feet

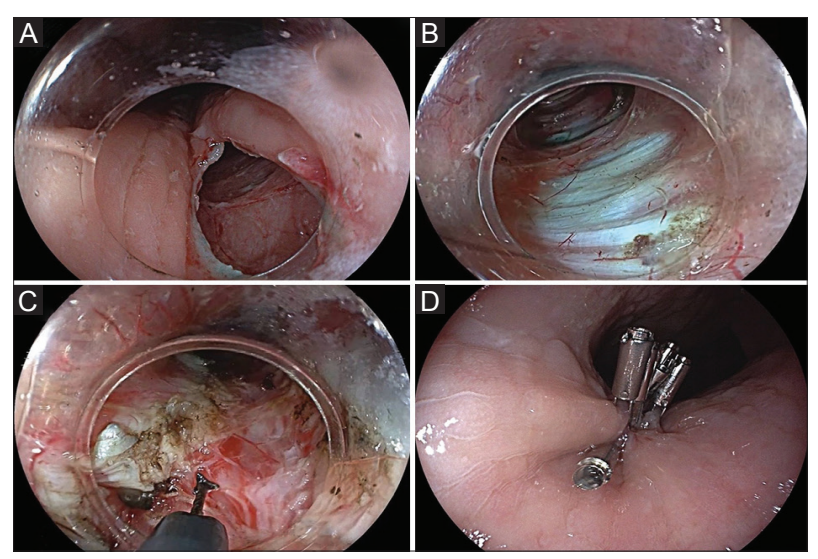

Figure 2 Endoscopic view of "posterior-like" anterior POEM at the 5-7 oclock position. (A) Mucosal entry. (B) Tunneling and exposure of the muscle layer. (C) Full-thickness myotomy. (D) Closure of the mucosal entry

\section{References}

1. Ramchandani M, Nabi Z, Reddy DN, et al. Outcomes of anterior myotomy versus posterior myotomy during POEM: a randomized pilot study. Endosc Int Open 2018;6:E190-E198.

2. Tan Y, Lv L, Wang X, et al. Efficacy of anterior versus posterior per-oral endoscopic myotomy for treating achalasia: a randomized, prospective study. Gastrointest Endosc 2018;88:46-54. 